\title{
Superhydrophobic Electrospun PVDF Membranes with Silanization and Fluorosilanization Co-Functionalized CNTs for Improved Direct Contact Membrane Distillation
}

\author{
Chongjie Gao ${ }^{\#}$, Wei Deng", Fuping Pan, Xuhui Feng and Ying $\mathrm{Li}^{*}$
}

Direct contact membrane distillation (DCMD) is a promising method for water purification and it is essential to fabricate membranes with high porosity, narrow pore distribution and high surface hydrophobicity. In this study, carbon-nanotubes (CNTs) with two surface functionalization, i.e., silanization and fluorosilanization, were compounded with polyvinylidene fluoride (PVDF) to prepare electrospun composite nanofiber membranes for DCMD. The effects of CNT surface functionalization on CNT dispersity and membrane pore size, porosity, hydrophobicity, and DCMD performance were investigated. The results showed that CNTs improved the membrane hydrophobicity and surface functionalization of CNTs furthered this improvement. It was found that membranes with co-functionalized CNTs achieved the highest distillate flux $\left(\sim 45 \mathrm{Lm}^{-2} \mathrm{~h}^{-1}\right)$ and salt rejection $(\sim 99.99 \%)$ among the tested membranes. This flux was $14 \%$ and $46 \%$ higher than the fluxes on the membranes with unfunctionalized-CNTs and without CNTs, respectively. Characterizations suggested that the superior performance was attributed to the co-functionalization improved dispersity of the CNTs, high porosity $(\sim$ $85.5 \%)$, large mean pore size $(\sim 0.89 \mu \mathrm{m})$ and surface superhydrophobicity (contact angle of $\left.\sim 153^{\circ}\right)$ of the membrane. This work implied that co-functionalization of CNTs is an avenue of great potential to improve the performance of PVDF membranes in DCMD application.

Keywords: Electrospinning; PVDF composite membranes; Surface functionalized CNTs; DCMD

Received 17 November 2019, Accepted 20 January 2020

DOI: $10.30919 / \mathrm{es} 8 \mathrm{~d} 905$

\section{Introduction}

Scarcity of clean water is one of the global issues that affect millions of people worldwide and this problem is becoming more and more severe due to population growth, industry development and climate change. ${ }^{1-3}$ To solve this problem, many advanced technologies have been developed, such as reverse osmosis (RO) and nanofiltration, but these technologies are still energy-consuming and costly, which blocks large-scale applications. ${ }^{4-9}$ Direct contact membrane distillation (DCMD) is a thermal-driven technology that uses the temperature difference to generate vapor pressure between the hot feed side and the cool distilled side across a hydrophobic membrane, consequently, water vapor transports through the membrane from the hot side and gets condensed on the cool side while salts and contaminants stay on the hot side (Fig. 3). As the working temperature of the feed side could be in the range of 30 to $90{ }^{\circ} \mathrm{C}$, lower than the boiling point of water, this technology can utilize the industrial waste heat, solar energy, and some renewable energy to heat the feed side, making it energy-conseving and economic-friendly. ${ }^{7,10-14}$

The membrane plays the most important role in the membrane distillation process since it is the determining part that sets a barrier to liquid water that contains salts and nonvolatile contaminants but allows clean water vapor to go through. Many membranes have been developed and studied, such as polypropylene (PP), polyvinylidene fluoride (PVDF),

J. Mike Walker '66 Department of Mechanical Engineering, Texas A\&M University, College Station, Texas, USA

${ }^{*}$ These authors contributed equally to this work.

*E-mail: yingli@tamu.edu (Y. Li) 
and polytetrafluoroethylene (PTFE) membranes. ${ }^{15}$ PVDF membranes are a promising candidate for DCMD because of the low cost, good chemical stability, and available large-scale fabrication. ${ }^{16,17} \mathrm{~A}$ lot of studies demonstrated that adding filler materials into the polymer matrix could modify the membrane characteristics, such as pore size, porosity, and surface hydrophobicity, so that to improve the DCMD performance. ${ }^{16-23}$ Carbon nanotubes (CNTs) have been extensively studied as a filler material for PVDF membranes because CNTs possess high aspect ratio, high thermal and mechanical stabilities, and light weight. ${ }^{7,1924-26}$ It has also been reported that surface functionalization, such as silanization and fluorosilanization, can further improve the dispersity of CNTs. For example, 3aminopropyltriethoxysliane (APTS) functionalized CNTs in PVDF membrane achieved $92.48 \%$ bovine serum albumin (BSA) rejection but only $64.2 \%$ for the nascent PVDF membrane, which was because APTS-induced silanization improved the dispersity of MWCNTs and compatibility between MWCNTs and polymer matrix and reduced the stress concentration points in the membranes. ${ }^{19,27}$ Fluorosilanization of CNTs could also improve the dispersity of CNTS and result in well-aligned CNTs inside PVDF crystalline fibers, as well as convey additional hydrophobicity. ${ }^{4,28,29}$ To our best knowledge, combination of silanization and fluorosilanization functionalization of CNTs for composite PVDF membranes to be used in DCMD has not been studied. We anticipate that the combination of these two types of surface functionalization will benefit both the morphology and hydrophobicity of the CNTs-PVDF membranes to achieve improved DCMD performance, outperforming membranes with single functionalization of the CNTs.

Electrospinning has received a wide attention in membrane manufacturing because electrospun membranes possess several attributes that make them attractive in separation technology, such as high porosity, interconnected open pore structure, pore sizes ranging from tens of nanometer to several micrometers, high permeability for gases, and a large specific surface area. ${ }^{30-34}$ Electrospun PVDF and PVDF-CNTs composite membranes with nanofiber structure have been extensively studied and applied for membrane distillation. ${ }^{7,18,29,32,34,35}$ \{Obaid, $2016 \# 25$ \} In this study, the combined silanization and fl uorosilanization method was utilized to modify the surface of CNTs. Functionalized CNTs were then mixed with PVDF to fabricate the composite membranes by the electrospinning method. The dispersity of the CNTs with and without functionalization was studied and membrane surface morphology, porosity, pore size, thickness, hydrophobicity and liquid entry pressure were characterized for commercial PVDF membrane, pure electrospun PVDF membrane, unfunctionalized-CNTs-PVDF and functionalizedCNTs-PVDFelectrospunmembraneforcomparison. Moreover, these membranes were tested in a DCMD setup and the performance, including flux and salt rejection, were studied. The possible role of functionalized CNTs in membrane distillation was also proposed.

\section{Materials and Experiments}

\subsection{Materials}

Polyvinylidene fluoride (PVDF) powder, Lithium Chloride, nitric acid, ethanol, N, N-dimethylformamide (DMF) and acetone were bought from VWR. Lithium Chloride was used as an additive for improving the conductivity of the polymer dope solution. Multi walled carbon nanotubes (CNTs) (average diameter: 6-9 nm, length: $5 \mu \mathrm{m},>99 \%$ ) were obtained from Sigma-Aldrich. F-127, $1 \mathrm{H}, 1 \mathrm{H}, 2 \mathrm{H}$, 2H-perfluorododecyl trichlorosilane (FTCS), octadecyltrichlorosilane (OTS) and toluene used for surface functionalization of CNTs and commercial PVDF membranes were bought from EMD, Millipore Corporation for reference. The chemical structures of OTS and FTCS are shown in Fig. 1. All these chemicals were used without further purification.
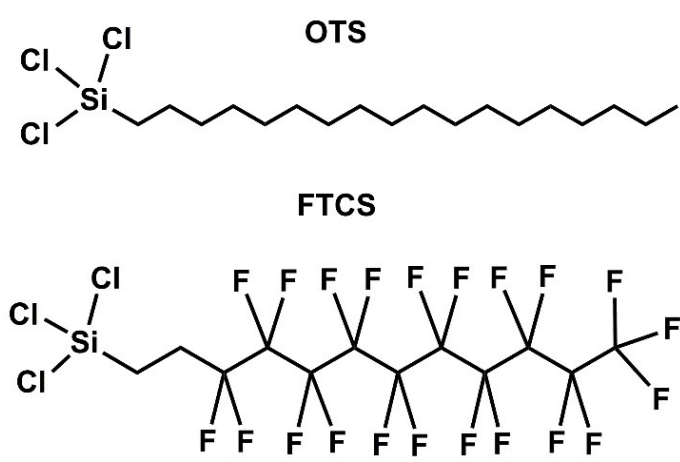

Fig. 1 Chemical structures of OTS and FTCS.

\subsection{Experiments}

\subsubsection{Pretreatment of CNTS}

$0.5 \mathrm{~g}$ CNTs was mixed with $80 \mathrm{~mL}$ concentrated nitric acid in sonication for $4 \mathrm{~h}$ and then the mixture was heated at $80^{\circ}$ $\mathrm{C}$ for $24 \mathrm{~h}$, aiming to get the carboxyl function groups on the surface of CNTs for further modification. After acid treatment, the CNTs was centrifuged at $12000 \mathrm{rpm}$ for 10 min and washed by 1 L DI water to remove the acid residual. Then CNTs was dried at $80{ }^{\circ} \mathrm{C}$ for $24 \mathrm{~h}$.

\subsubsection{Silanization Modification of CNTs}

$0.5 \mathrm{~g}$ pretreated CNTs and $50 \mathrm{~mL}$ toluene were mixed and sonicated for $2 \mathrm{~h}$ and then $20 \mathrm{~mL} 0.5 \mathrm{wt} \%$ OTS toluene solution was added into the mixture. The reaction was proceeded at $110{ }^{\circ} \mathrm{C}$ for $24 \mathrm{~h}$. The resultant OTS-CNTs were centrifuged and washed by both toluene and ethanol for three times and dried at $80{ }^{\circ} \mathrm{C}$ for $6 \mathrm{~h}$. 


\subsubsection{Fluorosilanization Modification of CNTs}

$0.5 \mathrm{~g}$ pretreated CNTs and $50 \mathrm{~mL}$ toluene were mixed and sonicated for $2 \mathrm{~h}$. After CNTs were well-dispersed, $20 \mathrm{~mL}$ $0.5 \mathrm{wt} \%$ FTCS toluene solution was added into the mixture. The mixture was then stirred for $24 \mathrm{~h}$. The final product was centrifuged, washed by toluene, and dried at $70^{\circ} \mathrm{C}$ for $6 \mathrm{~h}$.

\subsubsection{Silanization and Fluorosilanization \\ Co-functionalization of CNTs}

Co-functionalized CNTs were prepared using the similar approaches described in the above sections. The CNTs samples with silanization, fluorosilanization, silanization followed by fluorosilanization, and fluorosilanization followed by silanization were labelled as S-CNTs, F-CNTs, SF-CNTs, and FS-CNTs, respectively. Unfunctionalized CNTs were labelled as CNTs. Possible reactions between CNTs and OTS/FTCS are illustrated in Fig. 2, where the trichlorosilane groups on OTS/FTCS react with the carboxyl groups on acid treated CNTs. ${ }^{29}$

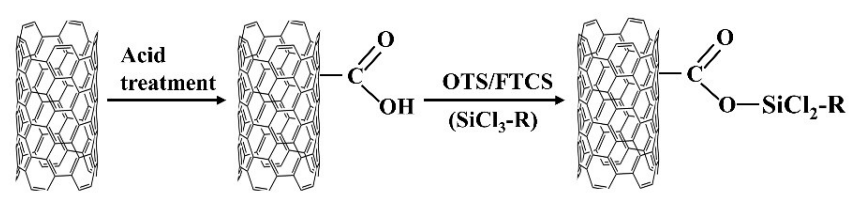

In OTS, $\mathrm{R}$ is $\left(\mathrm{CH}_{2}\right)_{17} \mathrm{CH}_{3}$; In FTCS, $\mathrm{R}$ is $\mathrm{CH}_{2} \mathrm{CH}_{2}\left(\mathrm{CF}_{2}\right)_{9} \mathrm{CF}_{3}$.

Fig. 2 Schematics of possible interactions between OTS/ FTCS and CNTs.

\subsection{Electrospinning of Nanocomposite Membranes}

PVDF powder was dissolved in a mixture of DMF and acetone (3:2 by weight ratio) at $50{ }^{\circ} \mathrm{C}$ for $12 \mathrm{~h}$ under magnetic stirring to get a homogeneous solution. Then functionalized and unfunctionalized CNTs and lithium chloride were added to the PVDF solution. The final weight concentration of PVDF, CNTs, and lithium chloride were $17 \%, 3 \%$, and $0.004 \%$, respectively. The metal needle used in the electrospinning process had an inner diameter of $0.377 \mathrm{~mm}$ and the feed rate was $0.9 \mathrm{~mL} / \mathrm{h}$. A metal rotating collector covered by the aluminum foil at a rotation speed of $500 \mathrm{rpm} / \mathrm{min}$ was used to collect the electrospun nanofibers. The voltage was adjusted to $20 \mathrm{kV}$. The distance between the collector and needle was $15 \mathrm{~cm}$. The environment humidity and temperature were $53 \pm 3 \%$ and $75 \pm 2{ }^{\circ} \mathrm{F}$, respectively. Moreover, pristine PVDF electrospun membranes were also fabricated under the same conditions.

\subsection{Characterization of Functionalized CNTs and Electrospun Membranes}

Fourier-transform infrared spectroscopy (FTIR) was performed on a Nicolet iS50 FT-IR spectrometer (Thermo Scientific) equipped with a liquid nitrogen cooled $\mathrm{HgCdTe}$ detector. The surface morphology was obtained by an ultra-high-resolution field-emission scanning electron microscope (FE-SEM, JEOL JSM7500F, Japan) equipped with a cold cathode UHV field emission conical anode gun. The porosity was measured by the gravimetric method. ${ }^{4}$ A $2 \mathrm{~cm} \times 2 \mathrm{~cm}$ sample was cut from the membrane and immersed into the absolute ethanol for $1 \mathrm{~h}$ to make sure that the membrane was fully wetted. The weight of membrane before and after wetted was measured. The membrane porosity $\varepsilon$ was calculated by Eq (1):

$$
\varepsilon=\frac{\left(m_{2}-m_{1}\right) \rho_{1}}{\left(\rho_{2}-\rho_{1}\right) m_{1}+\rho_{1} m_{2}}
$$

where $\rho_{1}$ is the density of PVDF, $\rho_{2}$ is the density of ethanol, $\mathrm{m}_{2}$ is the weight of the wetted membrane, and $\mathrm{m}_{1}$ is the weight of the dry membrane.

The mean pore size, maximum pore size, nanofiber diameter, and nanofiber diameter distribution were measured by analysis on SEM images using ImageJ. The thickness of membrane was measured by a micrometer caliper that has an accuracy of $1 \mu \mathrm{m}$ and 5 different positions were measured for each membrane to get the average value. The liquid entry pressure (LEP), which is the minimum pressure that makes liquid water permeate the membrane, was measured on a homemade setup. ${ }^{36}$ The hydrophobicity of the membranes was evaluated by water contact angles. The water contact angles were measured by an optical digital camera equipped with a stage and photograph analysis software. A $5 \mu \mathrm{L}$ of DI water droplet was dropped on the membrane surface carefully and the camera took photos to analyze the contact angle. The water contact angles were measured 5 times for each sample and then calculated to get the average value. ${ }^{37}$

\subsection{DCMD Tests}

The DCMD performance was tested by a laboratory scale setup with a $42 \mathrm{~cm}^{2}$ membrane area (CF042, Sterlitech, USA) illustrated in Fig. 3. All the tubes in the setup were insulated by insulation tapes to reduce heat loss. A $3.5 \%$ $\mathrm{NaCl}$ solution was applied in the feed side and DI water was used in the distillated side. The feed solution was maintained at $60^{\circ} \mathrm{C}$ by a hotplate and the distillate was maintained at $20^{\circ} \mathrm{C}$ by a chiller. The distillate tank was place on a digital balance and firstly filled with $300 \mathrm{~mL}$ DI water. A conductivity meter was used to measure the distillate conductivity and temperature. The data was recorded every half an hour. Water flux $J\left(\mathrm{Lm}^{-2} \mathrm{~h}^{-1}, \mathrm{LMH}\right)$ was calculated by Eq (2): 


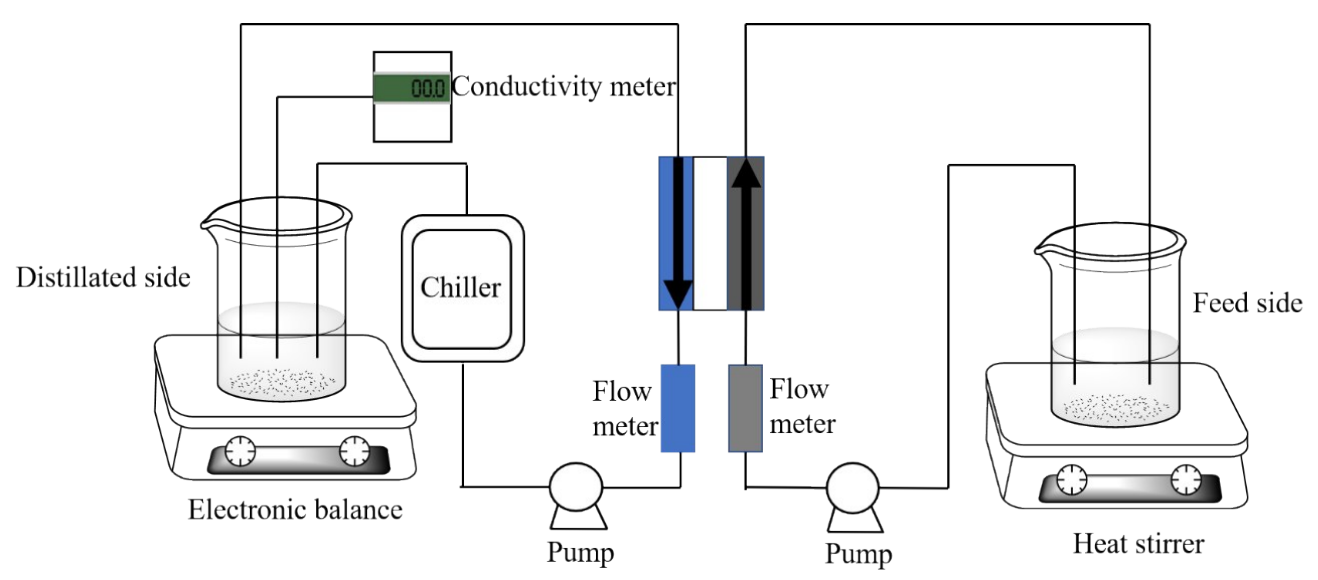

Fig. 3 Schematic diagram of the laboratory DCMD setup.

$$
J=\frac{\Delta m}{\rho A \Delta t}
$$

where $\Delta m$ was the weight gain at the distillated side, $\rho$ was the density of distillated solution, $A$ was the direct contact area of the membranes and $\Delta t$ was the time interval. The salt rejection efficiency (SR \%) was calculated by the ratio of distillate conductivity $(C)$ and initial conductivity of the feed solution $\left(C_{o}\right)$ using Eq (3).

$$
\operatorname{SR}(\%)=\left(1-\frac{C}{C_{0}}\right) \times 100 \%
$$

\section{Result and Discussion}

\subsection{Characterization of the CNTs}

FTIR analysis was performed to confirm that OTS and FTCS were successfully grafted onto the corresponding CNTs. The FTIR spectra of pristine CNTs, S-CNTs, FCNTs, SF-CNTs, and FS-CNTs were shown in Fig. 4. The absorption bands observed on all the samples at $1639 \mathrm{~cm}^{-1}$ and $1400 \mathrm{~cm}^{-1}$ are ascribed to the $\mathrm{O}-\mathrm{H}$ bending vibrations of chemically adsorbed water and $\mathrm{CH}_{2}$ bending, respectively. ${ }^{35,38}$ The spectra of modified CNTs are obviously different from the spectra of pristine CNTs from 1000 to $1200 \mathrm{~cm}^{-1}$; the spectra of modified CNTs have a broad peak in this range, which could be caused by the coexistence of the peak at $1080 \mathrm{~cm}^{-1}$ and $1180 \mathrm{~cm}^{-1}$. The bands around $1080 \mathrm{~cm}^{-1}$ are from the Si-O-Si bond, which indicated that the surface functionalization agents (FTCS and OTS) have successfully grafted on the surfaces of CNTs. ${ }^{39}$ The peaks at $1270 \mathrm{~cm}^{-1}$ are related to the C-F bond stretching, which confirmed that FTCS was introduced into F-CNTs, SF-CNTs and FS-CNTs. ${ }^{35}$ Therefore, the intended surface functionalization on CNTs was attained.

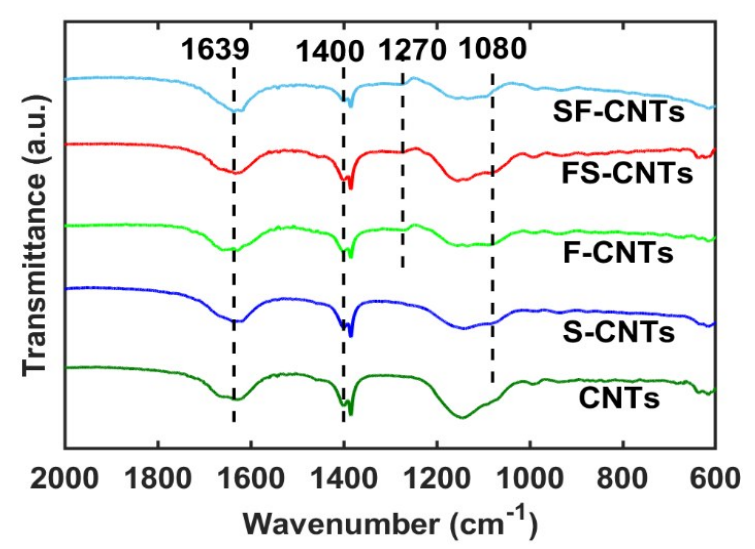

Fig. 4 FTIR spectra of functionalized and unfunctionalized CNTs.

In order to achieve uniform dispersion of the CNTs in PVDF matrix, it is important to exam the effects of silanization and fluorosilanization on the dispersity of CNTs in DMF-acetone mixtures (DMF: acetone $=3: 2$ ) that were also used as solvents for preparing PVDF dope solution. To do this, different CNTs samples were dispersed in the mixed solvents using sonication for $30 \mathrm{~min}$ and then the dispersions were placed still to check sedimentation. It can be seen in Fig. 5 that pristine CNTs and S-CNTs settled down quickly. After $1 \mathrm{~h}$, the supernatants became clear, indicating that pristine CNTs had unsatisfied dispersity in the mixed solution due to intrinsic van der Waals forces and this could not be remedied by surface silanization. Nevertheless, F-CNTs, SF-CNTs and FS-CNTs remained well suspended even after $24 \mathrm{~h}$ in the solutions. This unambiguously demonstrated that fluorosilanization could improve the suspension stability of CNTs in the DMFacetone solution. The use of fluorine containing groups for surface modification has been well applied to improve the dispersity of nanoparticles by minimizing surface energy. ${ }^{40}$ 
Positioning of highly electronegative fluorine at the end of the groups creates a partially negative charge for repulsive effects to prevent agglomeration. Entangled CNTs bundles could affect the stretching of the dope solution during the electrospinning process and lead to non-uniform porous structure. While modified CNTs have good dispersity that ensures well dispersed CNTs embedding nicely in the polymer matrix to help produce uniform porous structures of the composite membranes. ${ }^{29}$

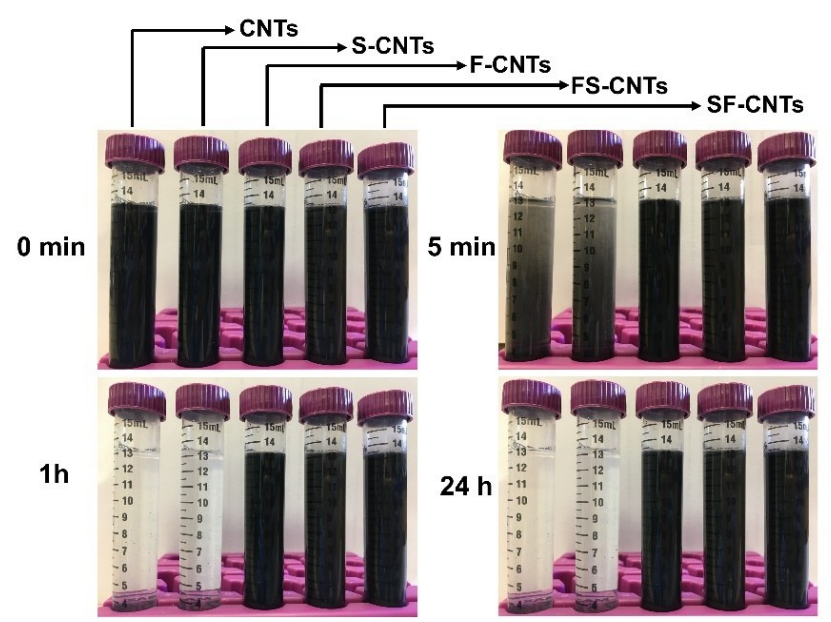

Fig. 5 Dispersity of functionalized and unfunctionalized CNTs in the DMF-acetone mixed solvents. Photographs were taken at $0 \mathrm{~min}, 5 \mathrm{~min}, 10 \mathrm{~min}, 1 \mathrm{~h}$ and $24 \mathrm{~h}$, respectively.

\subsection{Characterization of Electrospun Membranes}

\subsubsection{Membrane Hydrophobicity}

The surface hydrophobicity of the membranes was characterized by water contact angles and the results are showed in Fig. 6. Water contact angles on the commercial membrane and electrospun pristine PVDF membrane are $122^{\circ}$ and $132^{\circ}$, respectively. Incorporation of CNTs improved the hydrophobicity of the PVDF membranes as the CNTs-PVDF membrane has a contact angle of $142^{\circ}$. Since the S-CNTs-PVDF membrane also has the same contact angle of $142^{\circ}$, it is likely that surface silanization of CNTs did not help improve the hydrophobicity of CNTs. However, fluorosilanization of CNTs increased the contact angle to $150^{\circ}$ of the F-CNTs-PVDF membrane. The reason is that fluorosilanization introduced many hydrophobic fluoride groups on the surface of CNTs. ${ }^{29}$ The SF-CNTsPVDF membrane has the highest water contact angle of 153 ${ }^{\circ}$. As shown in Fig. 1, OTS and FTCS have similar chemical structure, that is a carbon chain with a $\mathrm{SiCl} 3$ group, but the carbon chain in OTS has 18 carbon atoms while the chain in FTCS has only 12 carbon atoms. This difference would make these chains have different lengths and make the cofunctionalized CNTs have higher surface roughness and high hydrophobicity than single functionalization. The FS-
CNTs-PVDF membrane, though also had co-functionalized CNTs, had a lower water contact angle $\left(\sim 148^{\circ}\right)$. This difference is possibly caused by the sequence of fluorosilanization and silanization on the CNTs; in the surface of FS-CNTs, some OTS chains might tangle with FTCS chains and blocked some fluoride groups, therefore, the effect of the hydrophobic fluoride group was inhibited, which did not occur on the surface of SF-CNTs where fluorosilanization was performed after silanization. Therefore, co-functionalization is superior to single functionalization in terms of improving surface hydrophobicity and the functionalization sequence matters.

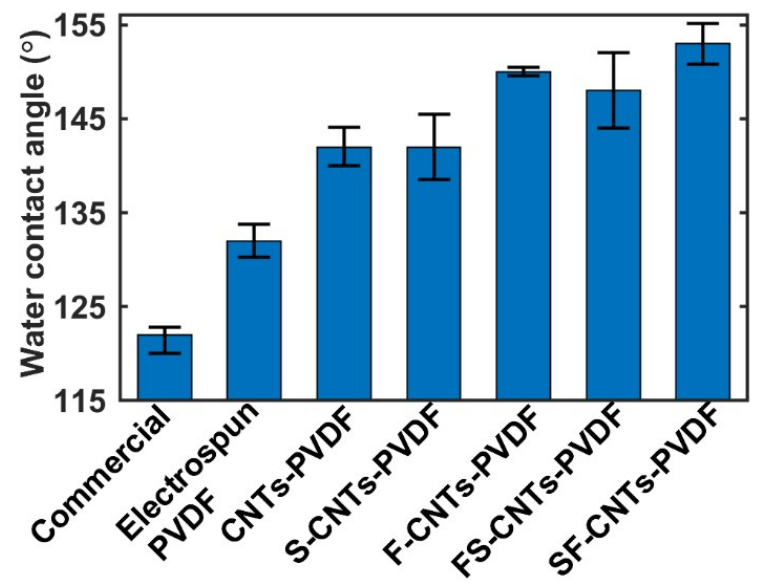

Fig. 6 Contact angles of water droplets on different membranes.

\subsubsection{Membrane Morphology and Liquid Entry Pressure}

The membrane morphology of the electrospun membranes was investigated by SEM. It can be seen in Fig. 7 that the membranes have typical interconnected nanofiber structure and there was no significant difference between the pure PVDF membrane and composite PVDF membranes regarding nanofiber morphology. The CNTs could not be observed mainly because most of the CNTs were embedded inside the nanofibers. ${ }^{29}$ The pore size, nanofiber diameter, and porosity of the membranes are summarized in Table 1 . The mean pore size of the composite membranes is around $0.9 \mu \mathrm{m}$, which is larger than that of pure PVDF membranes $(\sim 0.6 \mu \mathrm{m})$ and commercial PVDF membrane $(\sim 0.2 \mu \mathrm{m})$, while the composite membranes have a smaller nanofiber diameter than the pure PVDF membrane. This can be explained by the fact that the addition of CNTs increased the electrical conductivity of the dope solution and facilitated the formation of thinner nanofibers. Though CNTs also increased the viscosity of the dope solution, the raise of electric force caused by increasing of conductivity was higher than the raise of surface tension from the increasing of viscosity, consequently, the addition of CNTs decreased the nanofiber diameter. ${ }^{7,29,41,42}$ It was also noted 
that the membranes with fluorosilanization-functionalized CNTs have slightly thinner nanofibers. As discussed above, adding CNTs could increase the electrical conductivity of the dope solution and thus decrease fiber diameter. Surface functionalization induced better dispersity of CNTs and avoided the formation of tangled CNTs bundled that could influence the extrusion of the dope solution during electrospinning. Therefore, this fluorosilanization-induced better dispersity of the CNTs possibly contributed to thinner nanofibers. In addition, the electrospun membranes were much thinner than the commercial membrane, which would benefit the DCMD process because water vapor will experience less resistance when traveling through thinner membranes. The porosity of electrospun membrane $(\sim 85 \%)$ is slightly higher than the commercial one $(\sim 75 \%)$ and this was attributed to the unique interconnected nanofiber structure of electrospun membranes. ${ }^{34}$

Liquid entry pressure (LEP), the pressure that enables liquid water to penetrate the membranes, is a key parameter to evaluate the wetting resistance of membranes. A higher LEP is beneficial for membrane distillation to ensure feed water will not penetrate the membranes and contaminate the distillate. According to the Cantor-Laplace equation, LEP can be calculated by $\beta \gamma \cos (\theta) / r_{\max }$, where $\beta$ is the geometry factor, $\gamma$ is the surface tension of water, $\theta$ is the water contact angle, $r_{\max }$ is the maximum diameter of pore size in the membrane. ${ }^{4,29}$ From this equation, LEP is positively related to the water contact angle, i.e., hydrophobicity and inversely related to the maximum pore size. The commercial membrane has a LEP of $\sim 120 \mathrm{KPa}$ that is almost twice that of the electrospun membranes (Table 1). This phenomenon was attributed to the fact that the maximum pore size of electrospun membranes was much larger than the commercial one, though the electrospun membranes are more hydrophobic (Fig. 6). The LEP of electrospun membranes is about $60 \mathrm{KPa}$ and it is high enough for DCMD operation.

\subsection{Direct Contact Membrane Distillation Tests}

Fig. 8a showed the DCMD performance of the membranes. A $3.5 \% \mathrm{NaCl}$ solution was applied as the feed solutions and seven membrane samples were tested for DCMD,

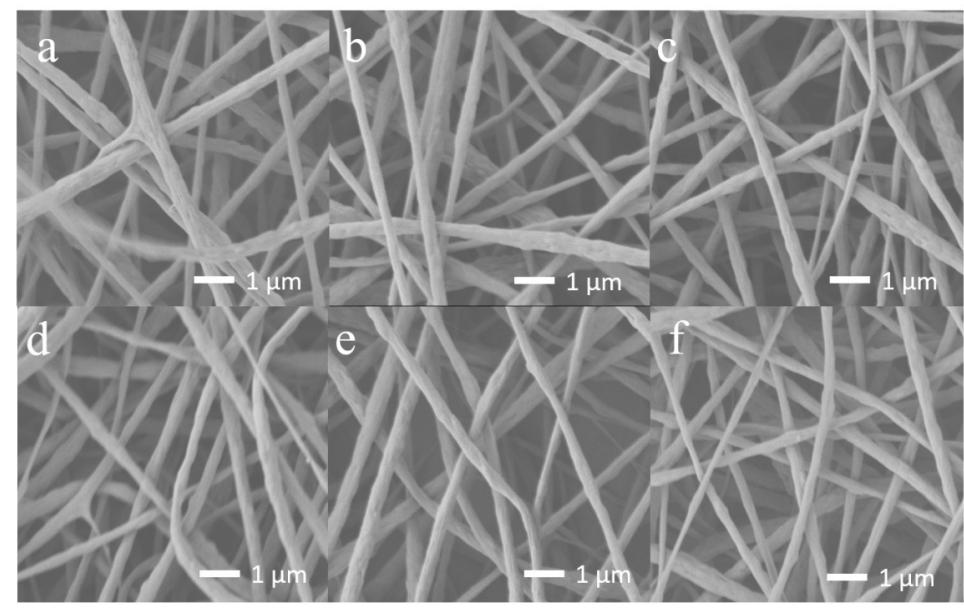

Fig. 7 SEM images of pristine PVDF membrane (a), CNTsPVDF membrane (b), S-CNTs-PVDF membrane (c), F-CNTsPVDF membrane (d), SF-CNTs-PVDF membrane (e), and FSCNTs-PVDF membrane (f).

Table 1 The characteristic parameters of electrospun and commercial PVDF membranes.

\begin{tabular}{ccccccc}
\hline Membrane & $\begin{array}{c}\text { Thickness } \\
(\mu \mathrm{m})\end{array}$ & $\begin{array}{c}\text { Porosity } \\
(\%)\end{array}$ & $\begin{array}{c}\text { Mean pore } \\
\text { size }(\mu \mathrm{m})\end{array}$ & $\begin{array}{c}\text { Max pore } \\
\text { size }(\mu \mathrm{m})\end{array}$ & $\begin{array}{c}\text { Mean fiber } \\
\text { diameter }(\mu \mathrm{m})\end{array}$ & $\begin{array}{c}\text { LEP } \\
(\mathrm{kPa})\end{array}$ \\
\hline Commercial & $125 \pm 4$ & $75.5 \pm 1.0$ & 0.22 & 0.40 & N/A & $120 \pm 1$ \\
Electrospun PVDF & $73 \pm 3$ & $87.3 \pm 0.4$ & 0.63 & 0.80 & $0.45 \pm 0.01$ & $65 \pm 7$ \\
CNTs-PVDF & $79 \pm 5$ & $85.3 \pm 3.6$ & 0.88 & 1.29 & $0.37 \pm 0.01$ & $63 \pm 2$ \\
S-CNTs-PVDF & $76 \pm 5$ & $84.2 \pm 2.1$ & 0.97 & 1.28 & $0.35 \pm 0.01$ & $56 \pm 2$ \\
F-CNTs-PVDF & $83 \pm 6$ & $84.0 \pm 4.9$ & 0.93 & 1.64 & $0.34 \pm 0.02$ & $60 \pm 5$ \\
SF-CNTs-PVDF & $86 \pm 1$ & $85.5 \pm 0.7$ & 0.89 & 1.54 & $0.34 \pm 0.03$ & $58 \pm 1$ \\
FS-CNTs-PVDF & $77 \pm 7$ & $83.7 \pm 0.9$ & 0.97 & 1.54 & $0.34 \pm 0.06$ & $68 \pm 6$ \\
\hline
\end{tabular}



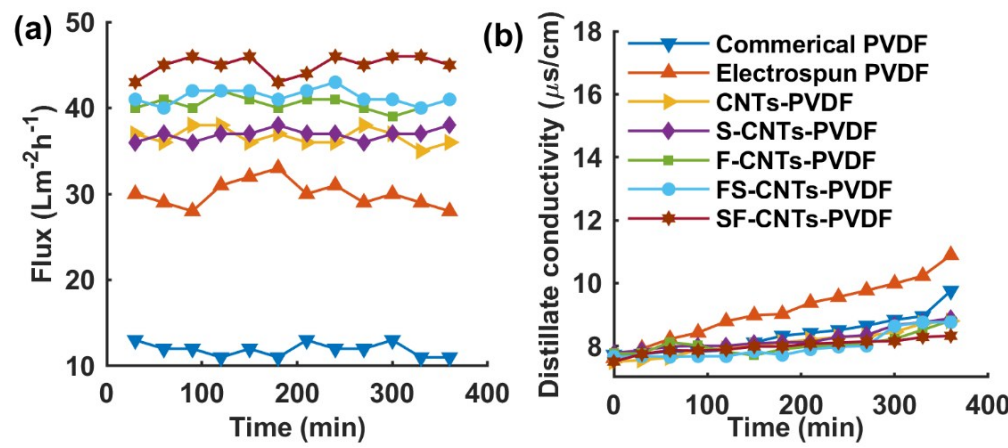

Fig. 8 Distillate flux (a) and conductivity (b) during the DCMD process.

including the commercial membrane, the electrospun pure PVDF membrane, CNTs-PVDF membrane, and four functionalized CNTs-PVDF membranes. The water flux of the commercial PVDF membrane was around $12 \mathrm{LMH}$ and remained stable during the $6 \mathrm{~h}$ test while the pure electrospun PVDF electrospun had a water flux around 30 $\mathrm{LMH}$, which was about 2.5 times that of the commercial one. This improvement could be explained from two aspects. First, the commercial PVDF membrane had a thickness of $125 \mu \mathrm{m}$ while the electrospun membranes were much thinner $(\sim 80 \mu \mathrm{m})$. The thinner electrospun membranes provided shorter water vapor diffusion distance and smaller resistance between the feed side and distillated side. Secondly, the porosity was $\sim 85 \%$ for the electrospun membranes but only $\sim 75 \%$ for the commercial one and the electrospun membranes had larger pore size, which made more water vapor pass through the membranes. The water fluxes of the CNTs-PVDF and S-CNTs-PVDF membranes were similar, both of which were about 36 LHM. This value was $20 \%$ higher than that of the electrospun pristine PVDF membrane. This improvement was probably a result of the increased mean pore size and max pore size of the CNTscontained PVDF membranes than the pure PVDF membrane. It was also proposed that some CNTs would form protrusions on the nanofiber surface that could further improve the surface roughness and water flux. Moreover, the surface of CNTs had many activated sites for adsorption and desorption of water vapor molecules that allowed a fast diffusion process for the vapor molecules. ${ }^{29,43}$ The reason that no significant flux difference was observed between the CNTs-PVDF and S-CNTs-PVDF membranes was probably that the two membranes had similar pore size (Table 1) and hydrophobicity (Fig. 6), which indicated that surface silanization for CNTs had limited improvement for PVDF membranes used in DCMD.

The F-CNTs-PVDF membrane achieved a flux of about 41 LMH and this was about $14 \%$ higher than those of the CNTsPVDF and S-CNTs-PVDF membranes, and $36 \%$ higher than that of the electrospun pure PVDF membrane. This should be due to the fact that fluorosilanization of the CNTs could improve the max pore size of the membranes (Table 1) and the hydrophobicity of membrane by grafting hydrophobic groups on the surface of CNTs. The highest flux was achieved by the SF-CNTs-PVDF membrane, which was approximately 45 LMH, clearly implying the benefits of silanization and fluorosilanization co-functionalization. The FS-CNTs-PVDF membrane, however, only had a flux about $42 \mathrm{LMH}$, smaller than that of the SF-CNTs-PVDF membrane but close to that of the F-CNTs-PVDF membrane. Due to different sequence of the two functionalization, there may be more hydrophobic groups on the surface of SF-CNTs than on the surface of FS-CNTs where silanization could diminish some fluorosilanizationgrafted hydrophobic groups since silanization was performed after fluorosilanization, resulting in better hydrophobicity of SF-CNTs-PVDF membranes than FS-CNTs-PVDF membranes.

Distillate conductivity and corresponding salt rejection were presented in Fig. $8 \mathrm{~b}$ and Fig. 9. The electrospun pure PVDF had slightly higher final distillate conductivity and lower salt rejection rate $(\sim 99.90 \%)$ than the commercial membrane $(\sim 99.92 \%)$. Other than that, all electrospun composite membranes achieved high salt rejection rate. Specifically, the SF-CNTs-PVDF membrane showed a salt rejection rate around $99.99 \%$. These results demonstrated the advantages of incorporating co-functionalized CNTs into electrospun PVDF membranes as both highest flux and salt rejection rate were obtained on the SF-CNTs-PVDF membrane.

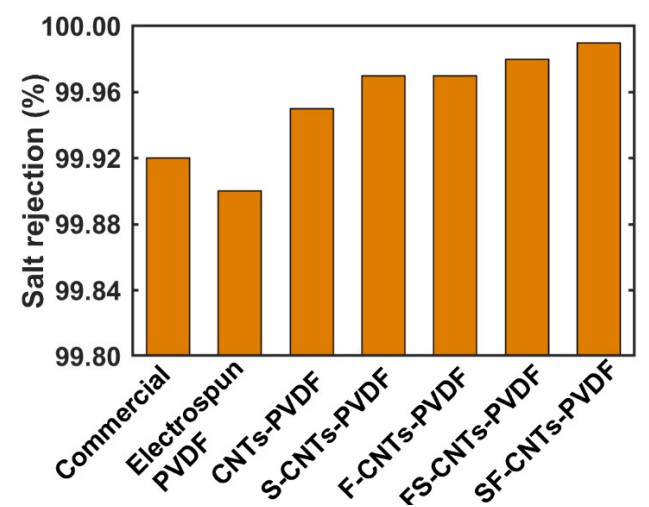

Fig. 9 Salt rejection efficiency of the membranes. 
3.4 Possible Mechanisms for Functionalized CNTsImproved DCMD Performance

Possible mechanisms of functionalized-CNTs in electrospun PVDF membranes improving DCMD performance were illustrated in Fig. 10. First, it has been reported that CNTs have very high rapid sorption and desorption capacity, providing water vapor diffusion pathways to go through the membrane and therefore benefitting vapor transportation. ${ }^{29,35,43}$ Secondly, surface fluorosilanization could reduce the surface energy and Van der Waals attraction between the CNTs, this would be helpful for improving the dispersion of CNTs in the polymer matrix and make full use of the functionalized CNTs. ${ }^{29,35}$ Thirdly, fluorosilanization functionalized-CNTs were highly hydrophobic and they could increase the surface roughness of the nanofibers via forming protrusions on the nanofiber surface. Fourthly, by combining surface silanization and fluorosilanization, two surface groups with different lengths of chains were introduced to CNTs surface, as shown in Fig. 10. These two different-length chains further increased the surface roughness of CNTs and nanofibers to improve the hydrophobicity. As a result, these co-functionalizedCNTs improved the hydrophobicity and wetting resistance of the membranes, which helped the superhydrophobic pore walls repel water vapor molecules to mitigate friction occurring between the pore walls and water vapor molecules to facilitate water vapor transportation. ${ }^{25,29,33}$

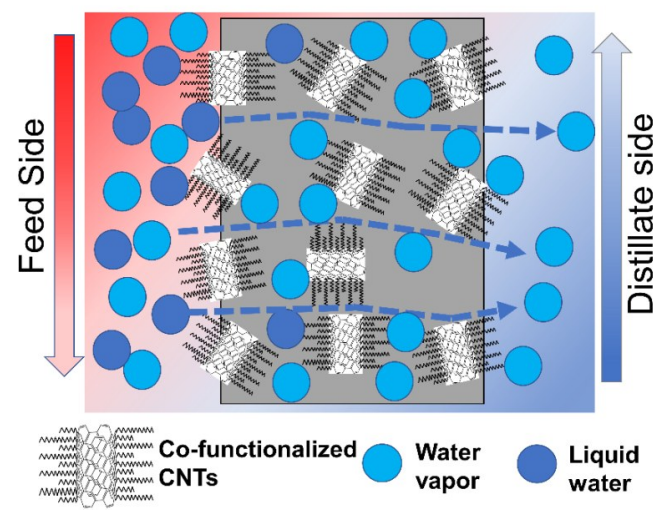

Fig. 10 Schematic diagram of possible mechanisms of functionalized-CNTs in electrospun PVDF membranes improving DCMD performance.

\section{Conclusion}

In this study, surface fluorosilanization and silanization were applied on CNTs and CNTs-PVDF composite membranes were fabricated via electrospinning and tested for DCMD. Highest surface hydrophobicity $\left(\sim 153^{\circ}\right)$, distillate flux $(45 \mathrm{LMH})$ and salt rejection rate $(\sim 99.99 \%)$ were achieved on the SF-CNTs-PVDF membrane that incorporated fluorosilanization and silanization cofunctionalized CNTs into the electrospun PVDF membrane.
The performance of this champion membrane was significantly superior to the commercial PVDF membrane, electrospun pure PVDF membrane and electrospun composite membranes that had single-functionalized CNTs. The great hydrophobicity of the SF-CNTs-PVDF membrane was attributed to the fluoride groups-grafted hydrophobic CNTs and CNTs-improved surface roughness. This outstanding hydrophobicity led to high rejection rate, while the high distillate flux was benefited from the high porosity, large pore size and small thickness. This work suggested that electrospun PVDF membranes with functionalized CNTs were promising candidates for membrane distillation and fluorosilanization-silanization co-functionalization on CNTs could be a potential avenue to modify CNTs for fabricating superior PVDF composite membranes.

\section{Notes}

The authors declare no competing financial interest.

\section{Acknowledgements}

This study was supported by Texas A\&M Energy Institute. The use of the Texas A\&M University Materials Characterization Facility is also acknowledged.

\section{References}

1. "Global Risks," World economic forum, 2015 - 10th Edition.

2. J. S. Famiglietti, Nat. Clim. Change, 2014, 4, 945.

3. M. A. Montgomery and M. Elimelech, Environ. Sci. Technol., 2007, 41, $17-$ 24.

4. L. F. Ren, F. Xia, V. Chen, J. Shao, R. Chen and Y. He, Desalination, 2017, 423, 1-11.

5. L. Eykens, K. De Sitter, C. Dotremont, L. Pinoy and B. Van der Bruggen, Ind. Eng. Chem. Res., 2016, 55, 9333-9343.

6. B. Peñate and L. García-Rodríguez, Desalination, 2012, 284, 1-8.

7. K. K. Yan, L. Jiao, S. Lin, X. Ji, Y. Lu and L. Zhang, Desalination, 2018, 437, 26-33.

8. B. Huo, D. Jiang, X. Cao, H. Liang, Z. Liu, C. Li and J. Liu, Carbon, 2019, 142, 13-19.

9. C. Li, D. Jiang, B. Huo, M. Ding, C. Huang, D. Jia, H. Li, C. Y. Liu and J. Liu, Nano Energy, 2019, 60, 841-849.

10. X. Li, X. Yu, C. Cheng, L. Deng, M. Wang and X. Wang, ACS Appl. Mater. Interfaces., 2015, 7, 21919-21930.

11. G. Rao and Y. Li, Desalin. Water Treat., 2016, 57, 21314-21327.

12. A. Khalifa, H. Ahmad, M. Antar, T. Laoui and M. Khayet, Desalination, 2017, 404, 22-34.

13. M. Qtaishat, T. Matsuura, B. Kruczek and M. Khayet, Desalination, 2008, 219, 272-292.

14. C. Li, W. Deng, C. Gao, X. Xiang, X. Feng, B. Batchelor and Y. Li, Sep. Purif. Technol., 2019, 224, 23-32.

15. M. Khayet, J. I. Mengual and T. Matsuura, J. Membr. Sci., 2005, 252, 101113.

16. D. Hou, J. Wang, D. Qu, Z. Luan and X. Ren, Sep. Purif. Technol., 2009, 69, 78-86.

17. Y. Liao, R. Wang, M. Tian, C. Qiu and A. G. Fane, J. Membr. Sci., 2013, 425, 30-39.

18. S. H. Wang, Y. Wan, B. Sun, L. Z. Liu and W. Xu, Nanoscale Res. Lett., $2014,9,522$. 
19. Z. Yu, G. Zeng, Y. Pan, L. Lv, H. Min, L. Zhang and Y. He, RSC Adv., 2015, 5, 75998-76006.

20. D. Hou, J. Wang, X. Sun, Z. Ji and Z. Luan, J. Membr. Sci., 2012, 405-406, 185-200.

21. J. A. Prince, G. Singh, D. Rana, T. Matsuura, V. Anbharasi and T. S. Shanmugasundaram, J. Membr. Sci., 2012, 397-398, 80-86.

22. S. Meng, Y. Ye, J. Mansouri and V. Chen, J. Membr. Sci., 2014, 463, $102-$ 112.

23. Y. Liao, R. Wang and A. G. Fane, Environ. Sci. Technol., 2014, 48, 63356341.

24. E. C. Mapunda, B. B. Mamba and T. A. Msagati, Phys. Chem. Earth, 2017, 100, $135-142$

25. T. L. Silva, S. Morales-Torres, J. L. Figueiredo and A. M. Silva, Desalination, 2015, 357, 233-245.

26. L. Zhao, W. Xu, J. Liu, W. Liu, J. Yao, M. Li, X. Wang and Y. Wu, J. SolGel Sci. Technol., 2014, 69, 107-113.

27. P. C. Ma, J. K. Kim and B. Z. Tang, Carbon, 2006, 44, 3232-3238.

28. A. Razmjou, E. Arifin, G. Dong, J. Mansouri and V. Chen, J. Membr. Sci., 2012, 415, 850-863.

29. A. K. An, E.J. Lee, J. Guo, S. Jeong, J. G. Lee and N. Ghaffour, Sci. Rep., 2017, 7, 41562.

30. Z. M. Huang, Y. Z. Zhang, M. Kotaki and S. Ramakrishna, Compos. Sci. Technol., 2003, 63, 2223-2253.

31. A. Haider, S. Haider and I. K. Kang, Arab. J. Chem., 2018, 18, 1165-1188.

32. Z. Zhao, J. Li, X. Yuan, X. Li, Y. Zhang and J. Sheng, J. Appl. Polym. Sci.,
2005, 97, 466-474

33. Y. C. Woo, L. D. Tijing, W. G. Shim, J. S. Choi, S. H. Kim, T. He, E. Drioli and H. K. Shon, J. Membr. Sci., 2016, 520, 99-110.

34. R. Gopal, S. Kaur, Z. Ma, C. Chan, S. Ramakrishna and T. Matsuura, J. Membr. Sci., 2006, 281, 581-586.

35. L. D. Tijing, Y. C. Woo, W. G. Shim, T. He, J. S. Choi, S. H. Kim and H. K. Shon, J. Membr. Sci., 2016, 502, 158-170.

36. K. Smolders and A. Franken, Desalination, 1989, 72, 249-262.

37. W. Deng, C. Li, F. Pan and Y. Li, Sep. Purif. Technol., 2019, 222, 35-44.

38. W. Deng, F. Pan, B. Batchelor, B. Jung, P. Zhang, A. Abdel-Wahab, H. Zhou and Y. Li, Environ. Sci-Wat. Res., 2019, 5, 769-781.

39. Z. Yuan, T. Nakamura, S. Adachi and K. Matsuishi, Nanoscale, 2017, 9 , 1193-1200.

40. X. Hou, J. He, L. Yu, Z. Li, Z. Zhang and P. Zhang, Appl. Surf. Sci., 2014, 316, 515-523.

41. Y. Liao, R. Wang, M. Tian, C. Qiu and A. G. Fane, J. Membr. Sci., 2013, 425426, 30-39.

42. L. D. Tijing, J. S. Choi, S. Lee, S. H. Kim and H. K. Shon, J. Membr. Sci, 2014, 453, 435-462.

43. K. Gethard, O. S. Khow and S. Mitra, ACS Appl. Mater. Interfaces, 2010, 3, 110-114.

Publisher's Note Engineered Science Publisher remains neutral with regard to jurisdictional claims in published maps and institutional affiliations. 